(95\% CI 91-94.7\%, p<0.0001) when both PLT and ALB were in normal range.

Conclusions US alone is not a reliable imaging modality for diagnosing cACLD. In suspected cases, TE should be carried out to determine the severity of hepatic fibrosis before a diagnosis of cACLD is made.

\section{PWE-42 MENTAL HEALTH READMISSIONS ARE INCREASED IN TRANSPLANT-FREE SURVIVORS OF ACUTE LIVER FAILURE}

${ }^{1}$ Mhairi C Donnelly*, ${ }^{2}$ Linda Williams, ${ }^{2}$ Nazir Lone, ${ }^{1}$ Kenneth J Simpson. ${ }^{1}$ Royal Infirmary of Edinburgh, Edinburgh, UK; ${ }^{2}$ University of Edinburgh, Edinburgh, UK

\subsection{6/gutjnl-2021-BSG.232}

Introduction Patients surviving acute liver failure (ALF) without transplantation (spontaneous survivors; SS) are at increased risk of later death and readmission to hospital. Most cases of ALF in Scotland are managed in the Scottish Liver Transplant Unit (SLTU) and are due to paracetamol overdose (POD). The aim of this work was to analyse mental health readmissions in Scottish ALF SS, compared with comparator cohorts.

Methods The index cohort (SLTU SS) consisted of patients admitted with severe acute liver injury or failure between 01/ $11 / 1992$ and 31/12/2014 who survived to hospital discharge without transplantation. Patients were identified from the SLTU ALF database. Control cohorts were: age, sex and postcode sector matched general population controls, patients admitted to hospital following POD in Scotland and not transferred to SLTU, patients admitted to Scottish intensive care units as non-surgical emergency admissions (SICSAG cohort) and patients surviving ALF with transplantation. Data related to mental health readmissions following discharge from the index admission were derived from the SMR04 database.

Results The SLTU SS cohort consisted of 708 patients $(80.2 \%$ POD). $25.6 \%$ of the SLTU SS had at least one mental health readmission $(29.5 \%$ of POD SS versus $10.0 \%$ of non-POD SS).

The SLTU SS were more likely to experience a mental health readmission compared with the matched control cohort (adjusted SHR 11.23, 95\% CI 8.55, 14.71), paracetamol overdose cohort (adjusted SHR 1.25, 95\%CI 1.04, 1.51) and the SICSAG cohort (adjusted SHR 2.76, 95\% CI 1.99, 3.82).

The adjusted relative rate of mental health readmissions was also higher in the SLTU SS compared with the matched control cohort $(10.63,95 \% 7.58,14.90)$, the paracetamol overdose cohort $(2.90,95 \%$ CI $2.23,3.77)$ and the SICSAG cohort $(5.91,95 \%$ CI $3.54,9.88)$. In the SLTU SS, POD aetiology and prior mental health admissions were predictors of later mental health readmissions.

The main psychiatric diagnosis in the SLTU SS was depressive disease (27.4\%) compared with alcohol misuse $(27.3 \%)$ in the matched controls, depressive disease (30.2\%) in the paracetamol overdose controls and alcohol misuse (32.1\%) in the SICSAG controls.

A comparative mental health morbidity analysis was not possible in the ALF survivors with transplant, as $<10$ posttransplant patients had any post-discharge mental health readmissions.

Conclusions Scottish ALF SS are at increased risk of mental health readmissions following discharge. The risk of readmission is higher in those with POD ALF compared with POD without ALF. Patients surviving ALF without transplant are currently discharged without follow up. This work suggests that psychiatric follow up -particularly in those with POD ALF - is warranted to reduce the burden of mental health readmissions.

\section{PWE-43 SARS-COV-2 ONE YEAR ON - THE WORRYING IMPACT ON EARLY DETECTION OF LIVER CANCERS}

1,2Daniel Geh*, 1,2Robyn Watson, 2,3Stuart McPherson, 2,35teven Masson, 2,3 Jessica Dyson, 2,3 Mhairi Donnelly, 2,3 Louise MacDougal, ${ }^{2,3}$ Preya Patel, ${ }^{3,4}$ John Hammond, ${ }^{3,5}$ Paul Turner, ${ }^{3,5}$ Tim Hoare, ${ }^{3,5}$ Kirsty Anderson, 3,5 Michael McNeil, ${ }^{3,4}$ Gourab Sen, ${ }^{3,4}$ Prof Steven White, ${ }^{3,4}$ Jeremy French, ${ }^{3,4}$ Stuart Robinson, Lucy Walker, ${ }^{1,2}$ Ms Misti McCain, ${ }^{6}$ Nick Wadd, ${ }^{7}$ Syed Asghar, ${ }^{3,8}$ Lavanya Mariappan, ${ }^{3,8}$ Jane Margetts, ${ }^{3}$ Yvonne Bury, ${ }^{3}$ Shreya Raman, ${ }^{3}$ Antony Darne, ${ }^{3}$ Beate Haugk, ${ }^{3,5}$ John Scott, ${ }^{3,5}$ Peter Littler, ${ }^{3,4}$ Derek M Manas, ${ }^{1,2,3}$ Helen L Reeves. 'Translational and Clinical Research institute, Newcastle University; ${ }^{2}$ Department of Medicine, Newcastle upon Tyne Hospitals NHS Foundation Trust; ${ }^{3}$ Hepatopancreatobiliary Multidisciplinary Team, Newcastle upon Tyne Hospitals NHS Foundation Trust; ${ }^{4}$ Department of Surgery, Newcastle upon Tyne Hospitals NHS Foundation Trust; ${ }^{5}$ Department of Radiology, Newcastle upon Tyne Hospitals NHS Foundation Trust; ${ }^{6}$ Department of Oncology, South Tees Hospital NHS Foundation Trust; ${ }^{7}$ Department of Oncology, Newcastle upon Tyne Hospitals NHS Foundation Trust; ${ }^{8}$ Department of Oncology, Cumberland Infirmary, North Cumbria Integrated Care NHS Foundation Trust

\subsection{6/gutjnl-2021-BSG.233}

Background In Northern England new primary liver cancer (PLC) diagnoses have increased by $10-15 \%$ year on year. We evaluated the impact of the SARS-CoV-2 pandemic.

Method In this retrospective, observational, regional study we evaluated 455 new PLC patients referred to the Newcastle-
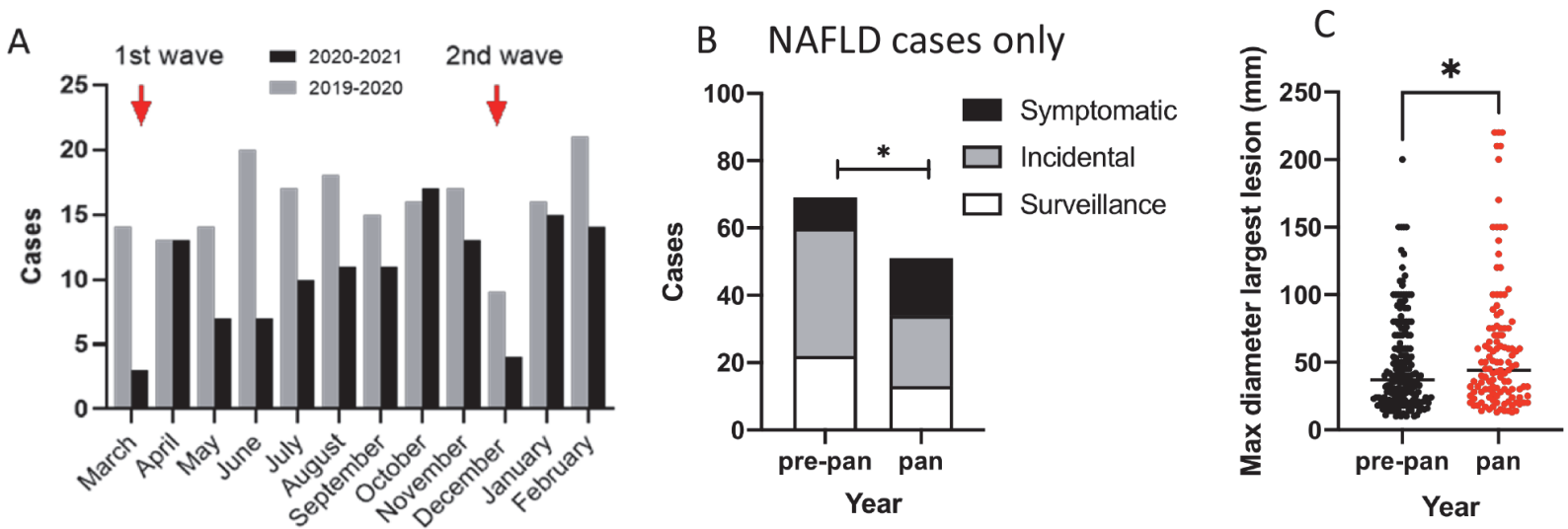

Abstract PWE-43 Figure 1 